\title{
Video Article \\ Brain State-dependent Brain Stimulation with Real-time Electroencephalography-Triggered Transcranial Magnetic Stimulation
}

\author{
Maria-loanna Stefanou ${ }^{1,2}$, David Baur ${ }^{1,2}$, Paolo Belardinelli ${ }^{1,2}$, Til Ole Bergmann ${ }^{1,2}$, Corinna Blum ${ }^{1,2}$, Pedro Caldana Gordon ${ }^{1,2}$, Jaakko \\ O. Nieminen ${ }^{1,2,3}$, Brigitte Zrenner ${ }^{1,2}$, Ulf Ziemann ${ }^{1,2}$, Christoph Zrenner ${ }^{1,2}$ \\ ${ }^{1}$ Department of Neurology \& Stroke, University of Tübingen \\ ${ }^{2}$ Hertie Institute for Clinical Brain Research, University of Tübingen \\ ${ }^{3}$ Department of Neuroscience and Biomedical Engineering, Aalto University
}

Correspondence to: Ulf Ziemann at ulf.ziemann@uni-tuebingen.de

URL: https://www.jove.com/video/59711

DOI: doi:10.3791/59711

Keywords: Behavior, Issue 150, real-time, brain state-dependent stimulation, EEG-TMS, corticospinal excitability, motor cortex, human brain plasticity, phase, oscillation

Date Published: 8/20/2019

Citation: Stefanou, M.I., Baur, D., Belardinelli, P., Bergmann, T.O., Blum, C., Gordon, P.C., Nieminen, J.O., Zrenner, B., Ziemann, U., Zrenner, C. Brain State-dependent Brain Stimulation with Real-time Electroencephalography-Triggered Transcranial Magnetic Stimulation. J. Vis. Exp. (150), e59711, doi:10.3791/59711 (2019).

\section{Abstract}

The effect of a stimulus to the brain depends not only on the parameters of the stimulus but also on the dynamics of brain activity at the time of the stimulation. The combination of electroencephalography (EEG) and transcranial magnetic stimulation (TMS) in a real-time brain statedependent stimulation system allows the study of relations of dynamics of brain activity, cortical excitability, and plasticity induction. Here, we demonstrate a newly developed method to synchronize the timing of brain stimulation with the phase of ongoing EEG oscillations using a real-time data analysis system. This real-time EEG-triggered TMS of the human motor cortex, when TMS is synchronized with the surface EEG negative peak of the sensorimotor $\mu$-alpha $(8-14 \mathrm{~Hz})$ rhythm, has shown differential corticospinal excitability and plasticity effects. The utilization of this method suggests that real-time information about the instantaneous brain state can be used for efficacious plasticity induction. Additionally, this approach enables personalized EEG-synchronized brain stimulation which may lead to the development of more effective therapeutic brain stimulation protocols.

\section{Video Link}

The video component of this article can be found at https://www.jove.com/video/59711/

\section{Introduction}

TMS is a well-established method for noninvasive brain stimulation and enables the specific modulation of ongoing network dynamics and studies of corticocortical and corticospinal neural pathways with high spatiotemporal precision ${ }^{1}$. When stimulating the primary motor cortex (M1), the neural response can be quantified as motor evoked potentials (MEPs), as well as TMS-evoked EEG potentials. MEPs can be recorded by electromyography (EMG) of target muscles, and their amplitude reflects corticospinal excitability when stimulating the primary motor cortex ${ }^{2}$.

Despite the unique potential of noninvasive brain stimulation as a scientific tool to investigate and modulate brain networks in healthy study participants and in patients, TMS studies suffer from large trial-to-trial and intra- and interindividual variability of evoked responses ${ }^{3,4,5}$. Specifically, in TMS studies of corticospinal excitability and plasticity, MEP responses, as well as induced long-term potentiation (LTP)- or longterm depression (LTD)-like plasticity, exhibit high intrinsic variability, even when the stimulus parameters are carefully controlled ${ }^{3,4}$. However, evidence from animal studies indicates that the observed variability of responses is not attributable to "random noise" but is instead related to the fluctuating brain states at the time of stimulation ${ }^{6}$. Accordingly, by combining TMS with EEG in a real-time brain-state-dependent stimulation paradigm (i.e., EEG-triggered TMS), the fluctuating instantaneous brain state can be used to optimize stimulus timing ${ }^{7,8,9,10}$.

Several studies have related the instantaneous phase of ongoing neural oscillations to neuronal excitability using TMS-compatible EEG systems $^{11,12}$. Modern EEG amplifiers can handle the large electromagnetic TMS artifacts, and increasingly well-established experimental protocols exist for the combination of EEG with TMS ${ }^{13,14}$ and the post hoc removal of TMS-related EEG artifacts ${ }^{15,16}$. While the influence of the prestimulus brain state as assessed by EEG on TMS-evoked responses can be assessed with randomly applied TMS stimuli that are sorted post hoc $^{17,18}$, the repetitive application of TMS in a predefined brain state requires real-time EEG-triggered TMS ${ }^{11,19}$.

Here, a custom millisecond-resolution EEG-triggered TMS setup is used to synchronize TMS pulses with a predetermined phase of ongoing brain oscillations ${ }^{11}$, demonstrating that the negative EEG deflection of the $\mu$-alpha rhythm corresponds to a higher cortical excitability state (leading to larger MEP amplitudes) as compared to the positive EEG deflection ${ }^{8,11,12,20}$. In this manuscript, we present a method for conducting real-time EEG-triggered TMS protocols to study human brain networks. 


\section{Protocol}

All experimental procedures described in the following sections have been approved by the Institutional Ethics Committee following the guidelines of the Declaration of Helsinki, and all participants provided written informed consent prior to study enrollment.

\section{Study Participants}

\section{Subject recruitment}

1. Recruit study participants based on predefined inclusion criteria. Screen candidates for contraindications, such as the presence of implanted medical devices (e.g., cardiac pacemaker), according to TMS safety guidelines ${ }^{21}$, or for neurological or psychiatric diseases and the use of drugs that act on the nervous system.

2. For studies requiring magnetic resonance imaging (MRI), assess the potential study participants for possible contraindications to MRI according to radiological safety standards ${ }^{22}$. Perform a power analysis to ensure that the study sample is sufficient for statistical analysis.

3. Optionally, preselect subjects having a prominent oscillation of interest in the signal extracted by the chosen EEG montage in order to improve the accuracy of the phase detection.

NOTE: In this experiment, the C3-centered Laplacian (C3 referenced to the average of the surrounding electrodes CP1, CP5, FC1, and FC5) was used to extract the sensorimotor $\mu$-rhythm with the subject at rest and eyes open. Preselected were subjects having a single peak in the alpha band $(8-14 \mathrm{~Hz}$ ) which contains $>25 \%$ of total power in the current source density (CSD) power spectrum. This criterion ensured that the oscillation amplitude was sufficiently large in comparison to the background noise (good signal-to-noise ratio [SNR]) to enable the algorithm to estimate the instantaneous phase of the trigger signal with sufficient accuracy and increased the likelihood of observing a significant excitability effect ${ }^{11,12,28,29,30}$.

\section{Subject information}

1. Provide the subjects with the study-related informed consent form. Provide printed TMS and MRI safety screening questionnaires. NOTE: These documents and the study protocol, as well as the use of personal data (e.g., from questionnaires) and identifiable human data (e.g., from MRI), need to be preapproved by the ethics committee (Institutional Review Board).

2. Ask the subject to fill out the TMS and MRI safety screening questionnaires. Acquire written informed consent for participation in the study and the planned use of data.

3. Acquire demographic data.

4. Assess subject handedness using standard inventories (e.g., the Edinburgh Handedness Inventory) ${ }^{23}$

5. Introduce the subject to the setup and stimulation procedure. Ensure that each participant is familiarized with the sensation of TMS and tolerates it well.

6. Acquire MRI for each participant prior to the TMS experimental sessions. Whole-head anatomical MR images are required, including the top of the scalp and anatomical landmarks (i.e., the tragus of both ears), as these will serve as fiducial points for neuronavigation in subsequent steps of this protocol.

7. Schedule the experimental sessions according to the specifications of the study protocol (i.e., take into account "washout periods" between the experiments).

NOTE: Ideally, subjects should come at the same time and on the same day of the week in protocols comparing different conditions in multiple sessions.

8. Instruct the participants to refrain from consuming alcohol, nicotine, or caffeine before the scheduled experimental sessions. The subjects should also have had their regular sleep on the night before the experiment and not be unusually tired.

\section{Setup Preparation}

1. Real-time-data-stream-capable EEG system

1. Use a TMS-compatible EEG/EMG amplifier that can handle the voltage spikes induced by the TMS pulse.

NOTE: The amplifier system needs to make a raw data stream available at a constant low latency ( $<5 \mathrm{~ms})$ for subsequent processing by a real-time processor. In this experiment, a 24-bit 80-channel biosignal amplifier was used for EEG and EMG recordings.

2. Configure the EEG/EMG amplifier system to low-pass filter (e.g., $0.16 \mathrm{~Hz}$ cut-off), and down-sample the biosignal data to $5 \mathrm{kHz}$ from the sampling rate at the amplifier head stage.

3. Ensure that the amplifier system sends data packets containing the relevant channels via a real-time user datagram protocol (UDP) to the real-time processor at regular constant intervals $\leq 1 \mathrm{~ms}$. Use a high sampling frequency (e.g., $5 \mathrm{kHz}$ ) to capture the EMG responses and to minimize filter delay of the EEG data.

2. EEG-compatible TMS device

1. Use a TMS device that can be triggered externally with a fixed and minimal delay and which minimizes artifacts in the simultaneous EEG recording (e.g., line noise in the EEG through the TMS coil cable, recharging artifacts after the pulse).

2. Ensure that the distance between the TMS stimulator (including the coil and coil cable) and the EEG recording system is maximized to reduce electrical interference (at least $1 \mathrm{~m}$ ). Where possible, turn off sources of electromagnetic interference such as fans and motors. Furthermore, ensure that the EEG and EMG recording leads are positioned and aligned such that common interference cancels out.

\section{Real-time EEG data processing system}

NOTE: The real-time EEG data stream is acquired and analyzed using a real-time digital signal processing system, which then triggers the TMS device when a predetermined condition is met. Such a system has been custom-developed in our laboratory ${ }^{11}$ to implement a phase detection algorithm similar to the approach by Chen et al. ${ }^{24}$ and consists of the following steps. 
1. Analyze a sliding window of data, $500 \mathrm{~ms}$ long (Figure 1a), to estimate the instantaneous phase of the target brain oscillation to phasespecifically trigger the TMS stimulator.

2. Perform bandpass filtering of the window for the frequencies of interest (e.g., between 9 and $14 \mathrm{~Hz}$ for the sensorimotor $\mu$-alpha rhythm; Figure 1b). Consider adjusting the filter parameters to the individual peak frequency of the target oscillation.

3. Remove any data distorted by the filtering edge effects. Note that there is a trade-off in that stronger filters have larger edge effects.

4. Use an autoregressive model to forward predict the signal (Yule-Walker, order 30; Figure 1c).

5. Apply a Hilbert transform of the resulting window of data to yield the analytic signal, from which the instantaneous phase of the signal is determined by taking the angle of the complex number at the relevant time-point.

6. Estimate the EEG power spectrum from the sliding window of data in the frequency bins of interest (e.g., 9-14 Hz) using a short-time Hann-windowed FFT.

7. When both phase and power meet a predetermined criterion (e.g., a negative peak, the minimum power threshold), generate a digital output (TTL) pulse with the real-time system to trigger the TMS device.

\section{Neuronavigation system}

1. To monitor coil position and achieve accurate and consistent TMS targeting within and across sessions, use a neuronavigation system. NOTE: A stereo infrared camera system is used precisely to locate in three-dimensional space reflective trackers, which are mounted on the subject's head and the stimulation coil, enabling precise relative positioning of the coil with respect to the individual's brain anatomy after calibration and MRI registration. For single-session studies and when planning to analyze only the EMG and not the EEG responses to TMS, navigation based on a standard brain instead of an individual MRI is sufficient.

2. Load the individual structural MRI data into the navigation system software prior to starting the experiment for each participant.

\section{Experimental control computer}

1. Use an experimental control computer that is connected to the EEG system, the TMS device, the real-time device, and the neuronavigation system.

NOTE: The EEG software controls the EEG amplifier system, sets parameters, and starts and stops EEG data archiving. The TMS device can be remote-controlled to change stimulation parameters (intensity, current direction, etc.) with a remote-control toolbox ${ }^{25}$.

2. Remotely control the real-time device to set the desired trigger conditions.

NOTE: The neuronavigation system can be remote-controlled, for example to target different coil locations.

3. Combine all of the above in an experimental control script to enable the automation of the experimental conditions and control flow.

\section{EEG recording electrodes}

1. Ensure that TMS-compatible EEG recording caps with the desired electrode layout are available in different sizes. Measure the subject's head circumference and prepare the appropriately sized cap.

2. Keep the required materials for EEG preparation handy (e.g., abrasive and conductive gels, syringes with sterile blunt needles, etc.).

\section{EMG recording electrodes}

1. Keep the surface EMG electrodes, leads, and required materials for skin preparation ready.

\section{Conducting the Experiment}

\section{Preliminaries}

1. Ensure that the required paperwork is in order (study consent form is signed) and that the participant has had no adverse effects since the previous session.

2. Seat the subject in a comfortable reclining position to minimize movement of the head during the experiment. A vacuum pillow wrapped around the neck and lower head can help to support the participant's head without causing additional muscle tension (e.g., like a chin rest would do)

\section{EEG and EMG preparation}

1. Place the appropriately sized EEG cap on the subject's head and position the cap correctly. Avoid excessive tension below the chin to reduce cranial and neck muscle activity that could contaminate the $\mathrm{EEG}^{26}$.

2. Register the subject in the EEG recording software.

3. Prepare the EEG electrodes according to the lab-specific protocol (e.g., apply abrasive gel followed by conductive gel).

4. Check that EEG electrode impedances are below $5 \mathrm{k} \Omega$.

5. To keep the conducting gel from drying up or getting smeared to the adjacent electrodes by any movement of the TMS coil, cover the EEG cap with plastic wrap. Then, fit a net cap above the plastic wrap to keep the cables in a fixed position to reduce EEG-artifact variability, and apply adhesive tape to increase the stability of the multiple layers.

6. Attach the surface EMG electrodes over the target muscles after having cleaned and lightly abraded the skin (e.g., use a bipolar recording from the right abductor pollicis brevis hand muscle in a belly-tendon montage). NOTE: Here, a bipolar recording from the right abductor pollicis brevis hand muscle in a belly-tendon montage was used. The placement of EMG electrodes is important as surface electrodes generally record activity from multiple underlying muscles.

7. Verify the correct matching between the actual EEG sensors on the head and the traces recorded in the EEG system by tapping on a few EEG electrodes to cause artifacts. As a sanity check, verify that occipital alpha increases when the participant closes their eyes.

8. Visually inspect the ongoing EEG and EMG signal for artifacts (e.g., line noise, muscle activity) or bad electrodes.

9. Ensure that the participant remains awake and keeps their eyes open throughout the experiment to avoid occipital alpha oscillations contaminating the signal.

\section{Preparation of the neuronavigation}

1. Attach the reflective head tracker to the participant's head with sufficient adhesive tape to ensure stability throughout the experiment. 
2. Use the pointer tool to coregister the head model with the relevant anatomical landmarks (e.g. the nasion, the tragi of both ears, the corners of the eyes).

3. Attach a coil tracker to the stimulation coil and calibrate the coil.

4. Place the pointer at different points on the head surface and verify the correctness of the displayed position on the monitor of the neuronavigation system.

5. Pinpoint the EEG sensor locations for coregistration with the individual MRI.

\section{Baseline EEG}

1. Demonstrate typical EEG artifacts to the subject (e.g., swallowing, chewing, eye blinks) and instruct the subject to avoid them throughout the experiment. Also, ask them to avoid jaw clenching, yawning, or talking.

2. Ask the subject to fixate on a point with the eyes open and perform a short recording of resting-state EEG with the eyes open.

3. If required for the computation of real-time filters, record additional EEG activity during tasks.

\section{Finding the motor "hotspot" and determination of the resting motor threshold}

1. Find the motor "hotspot" (i.e. the stimulation location over which single-pulse TMS elicits well-shaped MEPs of a comparably consistent amplitude across trials) and save the corresponding coil position (including coil orientation and angulation) in the neuronavigation system.

2. Find the resting motor threshold (RMT) by applying single TMS pulses over the motor cortex at gradually increasing stimulation intensities until the elicited MEPs have peak-to-peak amplitudes greater than $50 \mu \mathrm{V}$ in more than $50 \%$ of the trials ${ }^{2 \uparrow}$.

3. If available, use an automated script for parameter estimation by sequential testing (PEST), for instance, following a maximum likelihood strategy ${ }^{27}$ which also provides an online estimate of the confidence interval of RMT based on the observed variability of single responses and which typically requires ca. 30 test pulses of adaptively varying intensity to obtain a robust RMT estimate.

4. If this is not the first experimental session, compare the coil position with the previous position and compare the obtained RMT with the previous RMT to validate consistency.

5. If required, determine stimulation intensities for the active motor threshold (AMT) or for the 1-mV peak-to-peak MEP amplitude using standard procedures ${ }^{21}$.

\section{Final participant preparation}

1. Optionally, immobilize the head of the subject using a vacuum pillow.

2. Optionally, deliver a masking noise through earplugs (when planning to analyze TMS-evoked EEG potentials). Otherwise, provide the subject with earplugs and headphones for hearing protection.

3. Optionally, align and fix the coil at the desired position using a mechanical arm.

\section{Pre-experiment data quality validation}

1. Check that the real-time processor is receiving data from the EEG system.

2. Check the signal obtained from the desired EEG spatial filter (e.g., C3-centered Laplacian montage) for obvious artifacts.

3. Visually confirm the EEG signal quality, check for bad electrodes, excessive line noise, and muscle artifacts, and adjust the time window and amplitude scaling on the EEG system software for ongoing visual inspection during the experiment.

\section{Main experimental session}

1. Unless the stimulator intensity is remote-controlled in the experimental script, manually set the stimulation intensity to the desired value (e.g., $110 \%$ of the RMT).

2. Start the experimental script to apply pulses at different phases of the target oscillation in a randomized order.

3. During the experiment, monitor the trigger condition thresholds (artifact-detection threshold, pre-innervation threshold, minimum power, etc.).

NOTE: Stimuli will be triggered at irregular intervals, as the real-time processor is waiting for the trigger conditions to occur. However, the conditions should be set such that most stimuli occur within a predictable interval (e.g., 2-3 s after the previous pulse), and long pauses (e.g., in this case, $>5 \mathrm{~s}$ ) are avoided as these would lead to larger evoked responses due to novelty.

1. Alternatively, use post hoc stratification to remove trials following overly long intervals.

4. To achieve sufficient statistical power to differentiate phase-specific stimulation effects, acquire a sufficient number of trials NOTE: We typically chose $80-120$ interleaved trials per condition ${ }^{20}$.

5. Document the start and end times of the various sessions and keep a record of any unusual occurrences.

\section{Representative Results}

The utilization of the real-time EEG-triggered TMS in the human primary motor cortex reveals differential corticospinal excitability and plasticity effects. Using the protocol described above, real-time EEG-TMS was applied, synchronizing TMS with the ongoing EEG oscillatory phase of the endogenous sensorimotor $\mu$-rhythm in three trigger conditions (positive peak, negative peak, and random phase) in randomized order. A Laplacian EEG montage was used to extract the sensorimotor $\mu$-rhythm by referencing the EEG electrode $\mathrm{C} 3$ to the average of four surrounding electrodes (FC1, FC5, CP1, and CP5). Figure 2a shows the average prestimulus EEG signal in the 400 ms before the TMS pulse for the three predefined conditions. The average elicited MEPs recorded from the right-hand muscles are depicted in Figure $\mathbf{2 b}$. These results show that the negative EEG deflection of the $\mu$-rhythm corresponds to a higher cortical excitability state (leading to larger MEP amplitudes) as compared to the positive EEG deflection, with low intertrial variability of the noted corticospinal excitability effects, presented in Figure 2c. 


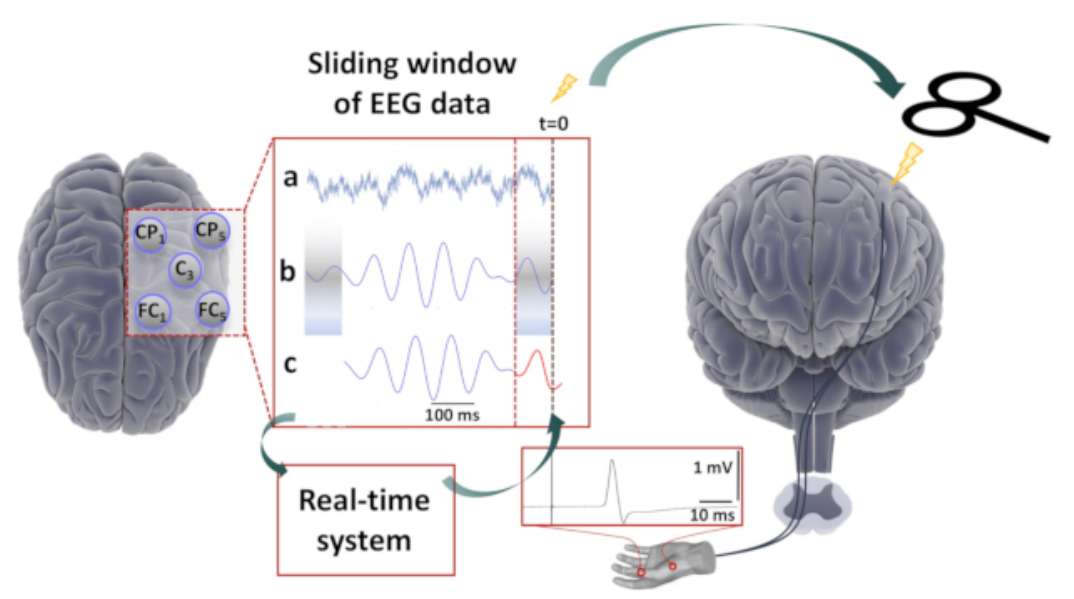

Figure 1: Brain-state-dependent EEG-phase-triggered TMS. Scalp EEG raw data derived from a five channel Laplacian montage centered on the C3 electrode over the left sensorimotor cortex was acquired sample-by-sample by a real-time digital signal processing system. (a) A 500ms sliding window of EEG data was processed by the algorithm every $2 \mathrm{~ms}$. (b) The signal after band-pass filtering and removal of the edge artifacts. (c) The forward-predicted signal (red trace) based on an autoregressive forecasting model that was calculated from the window of data. The phase at time zero ("right now") was estimated using a Hilbert transform, the spectral power was estimated from the window of data. The TMS stimulator was triggered when a predefined phase and spectral amplitude condition were met. TMS over the left primary motor cortex resulted in MEPs in right-hand muscles recorded with surface EMG. Please click here to view a larger version of this figure.

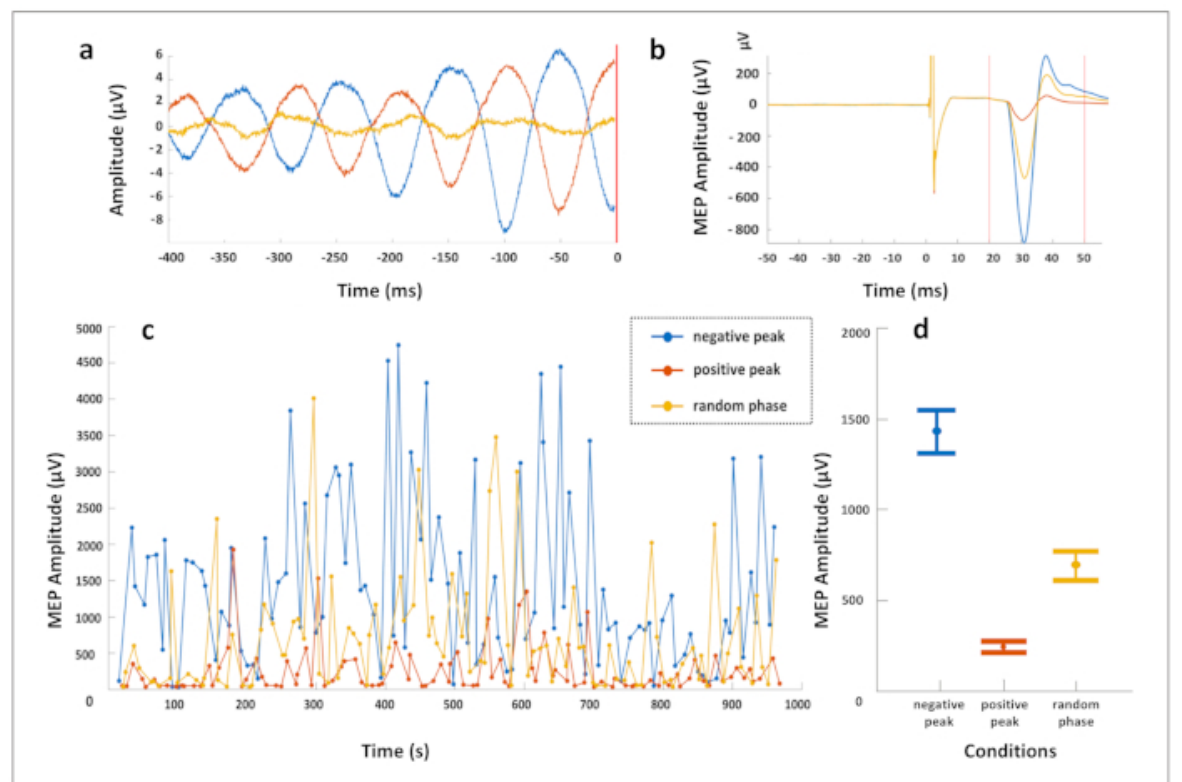

Figure 2: Data from one exemplary subject who received real-time EEG-triggered TMS over the left M1, targeting the phase of the 10 Hz sensorimotor $\mu$-rhythm. A hundred stimuli each were applied according to three phase-trigger conditions (positive peak, negative peak, and random phase) in combination with a constant minimum $10 \mathrm{~Hz}$ spectral power threshold condition, in randomized order, with an intertrial interval of approximately $3 \mathrm{~s}$. A Laplacian EEG montage was used to extract the sensorimotor $\mu$-rhythm by referencing the EEG electrode C3 to the average of four surrounding electrodes (FC1, FC5, CP1, and CP5). (a) Average prestimulus EEG signal in the $400 \mathrm{~ms}$ before the TMS pulse for the three conditions. (b) Average EMG trace of the motor evoked potential (MEP) recorded from the right abductor pollicis brevis muscle for each condition. (c) Peak-to-peak MEP amplitude (in microvolts) of each trial over time, per trigger condition. Note that the MEPs are largest in the negative peak condition, smallest in the positive peak condition, and intermediate in the random phase condition. (d) The mean MEP amplitude in each condition is shown with error bars illustrating the standard error of the mean. Note that a participant with a particularly clear effect has been selected for illustration purposes and that this effect size is not representative for the group average. Please click here to view a larger version of this figure.

\section{Discussion}

Brain-state-dependent EEG-triggered TMS is a novel method with unique perspectives with respect to effectiveness and consistency of the ensuing brain-stimulation effects ${ }^{8,9,31}$. The main advantage of the method is that a functionally relevant endogenous brain state may be specifically targeted to trigger the TMS pulse, inducing potentially less variable and longer-lasting brain responses ${ }^{11}$. Real-time EEG-triggered 
repetitive TMS in the negative phase of the sensorimotor $\mu$-rhythm of human M1 (i.e., the state of increased corticospinal excitability, Figure 2) induced significantly stronger LTP-like plasticity (a long-term increase of MEP amplitude) compared to brain-state-independent TMS ${ }^{11,20}$. In addition to its scientific utility, the application of real-time EEG-TMS to cortical areas, such as the dorsolateral prefrontal cortex (DLPFC), has the potential to increase the effectiveness of current therapeutic brain stimulation protocols.

In this manuscript, we presented the methodological steps for the implementation of real-time EEG-TMS. Fundamental requirements for the conduction of experiments with this method are, first, the use of a TMS-compatible EEG system with a real-time digital out option and, second, the use of real-time signal processing with the implementation of a phase-detection algorithm ${ }^{24}$, which extracts the desired brain rhythm (e.g., sensorimotor $\mu$-rhythm) from the recorded EEG signal using spatial filters (e.g., C3-centered Laplacian filter) and applies stimulation when preselected conditions (i.e., phase and power of the targeted brain rhythm) are met. The performance and accuracy of the algorithm depend strongly on the SNR of the EEG recording ${ }^{20}$. Thus, the EEG preparation steps of the protocol are crucial to achieve a high SNR and ensure accurate triggering of the TMS, and a preselection of participants may need to be considered if the respective target oscillation is not sufficiently observable with EEG in every individual. Furthermore, the use of mechanical support arms for the coils and vacuum pillows to immobilize the participant's head is advisable, in order to minimize artifacts due to the varying pressure of the coil on the electrodes.

Regarding the application of the real-time EEG-TMS method in experimental paradigms, the selection of the brain rhythm of interest may vary. Thus, adjustments of the filtering are advisable to facilitate the identification of the targeted brain activity. Recently, several spatial filtering methods have been proposed to optimally extract a functionally relevant brain state (e.g., in channel space ${ }^{19}$, with current source density ${ }^{13}$, with local spatial filters ${ }^{11,28}$, and with individualized filters using, for example, spatial-spectral decomposition ${ }^{29}$ ). Yet, so far, no unequivocal method exists to extract from surface EEG signals (sensor space) the real brain-oscillation phase (source space). Future studies that assess the correspondence of surface and source-space signals are warranted to improve the precision of real-time EEG algorithms.

Whereas in this protocol we have focused on the $8-14-\mathrm{Hz}$ sensorimotor $\mu$-rhythm to demonstrate the influence of the instantaneous phase of this oscillation on corticospinal excitability, other oscillations (e.g., beta, theta, or infraslow oscillations) may also play a role. This method can, in principle, be used to target the phase for any oscillation that can be isolated with a sufficient SNR, including multiple superimposed oscillations (e.g., a negative cycle of alpha and a simultaneous positive peak of gamma).

One main limitation of the real-time EEG-TMS experiments is that the spatiotemporal resolution with respect to the brain sources is strongly dependent on artifact occurrence and consistency of the stimulation. Therefore, a critical prerequisite of the protocol is the monitoring of the performance of the algorithm (i.e., ensuring that stimulation occurs upon the detection of neuronal and not artifactual activity throughout the experiment). Furthermore, the utilization of neuronavigation for optimal and consistent positioning of the stimulation coil (especially in experimental paradigms using stimulation sites such as the DLPFC) is helpful for reducing response variability due to variability in coil position. Note also, as a further limitation, that specifically selected and configured EEG/EMG, TMS, and real-time processing devices are required, along with experience in preparing and conducting the experiments in such a way as to minimize external sources of response variability that may mask the effect of instantaneous brain-state.

In conclusion, we demonstrated a standard protocol for conducting real-time EEG-TMS experiments and introduced a novel method for utilizing the endogenous brain states of interest (i.e., preselected phases and power of a targeted endogenous brain oscillation) to trigger brain stimulation. Further research using the real-time EEG-TMS method will allow methodological improvements and facilitate the development of effective protocols for the study and modulation of human brain networks.

\section{Disclosures}

C.Z. and P.C.G. are partially funded through an EXIST Transfer of Research grant by the German Federal Ministry for Economic Affairs and Energy (grant 03EFJBW169). C.Z. reports additional employment as a part-time employee of the not-for-profit medical innovation foundation (Stiftung für Medizininnovationen, Tübingen, Germany); a subsidiary of this foundation is producing the real-time processor used in this article (Medical Innovations Incubator $\mathrm{GmbH}$, Tübingen, Germany).

\section{Acknowledgments}

C.Z. acknowledges support from the Clinician Scientist Program of the Faculty of Medicine, University of Tübingen. U.Z. acknowledges support from the German Research Foundation (grant ZI 542/7-1). T.O.B. acknowledges support from the German Research Foundation (grant BE 6091/2-1). J.O.N. acknowledges support from the Academy of Finland (Decisions No. 294625 and 306845). The authors acknowledge support by the Open Access Publishing Fund of the University of Tübingen.

\section{References}

1. Hallett, M. Transcranial magnetic stimulation: a primer. Neuron. 55(2), 187-199 (2007).

2. Barker, A. T., Jalinous, R., Freeston, I. L. Non-invasive magnetic stimulation of human motor cortex. Lancet. 1(8437), 1106-1107 (1985).

3. López-Alonso, V., Cheeran, B., Río-Rodríguez, D., Fernández-del-Olmo, M. Inter-individual variability in response to non-invasive brain stimulation paradigms. Brain Stimulation. 7(3), 372-380 (2014).

4. Müller-Dahlhaus, J. F., Orekhov, Y., Liu, Y., Ziemann, U. Interindividual variability and age-dependency of motor cortical plasticity induced by paired associative stimulation. Experimental Brain Research. 187(3), 467-475 (2008).

5. Ziemann, U., Siebner, H. R. "Inter-subject and inter-session variability of plasticity induction by non-invasive brain stimulation: Boon or bane? Brain Stimulation. 8(3), 662-663 (2015).

6. Arieli, A., Sterkin, A., Grinvald, A., Aertsen, A. Dynamics of ongoing activity: explanation of the large variability in evoked cortical responses. Science. 273(5283), 1868-1871 (1996). 
7. Thut, G., Ives, J. R., Kampmann, F., Pastor, M. A., Pascual-Leone, A. A new device and protocol for combining TMS and online recordings of EEG and evoked potentials. Journal of Neuroscience Methods. 141(2), 207-217 (2005).

8. Zrenner, C., Belardinelli, P., Müller-Dahlhaus, F., Ziemann, U. Closed-loop neuroscience and non-invasive brain stimulation: A tale of two loops. Frontiers in Cellular Neuroscience. 10, 92 (2016).

9. Bergmann, T. O. Brain state-dependent brain stimulation. Frontiers in Psychology. 9, 2108 (2018).

10. Matthews, P. B. C. The effect of firing on the excitability of a model motoneurone and its implications for cortical stimulation. Journal of Physiology. 518(3), 867-882 (1999).

11. Zrenner, C., Desideri, D., Belardinelli, P., Ziemann, U. Real-time EEG-defined excitability states determine efficacy of TMS-induced plasticity in human motor cortex. Brain Stimulation. 11(2), 374-389 (2018).

12. Stefanou, M. I., Desideri, D., Belardinelli, P., Zrenner, C., Ziemann, U. Phase synchronicity of $\mu$-rhythm determines efficacy of interhemispheric communication between human motor cortices. Journal of Neuroscience. 38(49), 10525-10534 (2018).

13. Berger, B., Minarik, T., Liuzzi, G., Hummel, F. C., Sauseng, P. EEG oscillatory phase-dependent markers of corticospinal excitability in the resting brain. BioMed Research International. 2014, 936096 (2014).

14. Keil, J. et al. Cortical brain states and corticospinal synchronization influence TMS-evoked motor potentials. Journal of Neurophysiology. 111(3), 513-519 (2014).

15. Rogasch, N. C. et al. Analysing concurrent transcranial magnetic stimulation and electroencephalographic data: A review and introduction to the open-source TESA software. Neurolmage. 147, 934-951 (2017).

16. Herring, J. D., Thut, G., Jensen, O., Bergmann, T. O. Attention modulates TMS-locked alpha oscillations in the visual cortex. Journal of Neuroscience. 35(43), 14435-14447 (2015).

17. Romei, V. et al. Spontaneous fluctuations in posterior a-band EEG activity reflect variability in excitability of human visual areas. Cerebral Cortex. 18(9), 2010-2018 (2008).

18. Sauseng, P., Klimesch, W., Gerloff, C., Hummel, F. C. Spontaneous locally restricted EEG alpha activity determines cortical excitability in the motor cortex. Neuropsychologia. 47(1), 284-288 (2009).

19. Bergmann, T. O. et al. EEG-guided transcranial magnetic stimulation reveals rapid shifts in motor cortical excitability during the human sleep slow oscillation. Journal of Neuroscience. 32(1), 243-253 (2012).

20. Schaworonkow, N., Triesch, J., Ziemann, U., Zrenner, C. EEG-triggered TMS reveals stronger brain state-dependent modulation of motor evoked potentials at weaker stimulation intensities. Brain Stimulation. 12(1), 110-118 (2019).

21. Rossi, S., Hallett, M., Rossini, P. M., Pascual-Leone, A., The Safety of TMS Consensus Group. Safety, ethical considerations, and application guidelines for the use of transcranial magnetic stimulation in clinical practice and research. Clinical Neurophysiology. 120(12), 2008-2039 (2009).

22. Kanal, E. et al. ACR guidance document for safe MR practices: 2007. American Journal of Roentgenology. 188(6), 1447-1474 (2007).

23. Oldfield, R. C. The assessment and analysis of handedness: The Edinburgh inventory. Neuropsychologia. 9(1), 97-113 (1971).

24. Chen, L. L., Madhavan, R., Rapoport, B. I., Anderson, W. S. Real-time brain oscillation detection and phase-locked stimulation using autoregressive spectral estimation and time-series forward prediction. IEEE Transactions on Biomedical Engineering. 60(3), 753-762 (2013).

25. Habibollahi Saatlou, F. et al. MAGIC: An open-source MATLAB toolbox for external control of transcranial magnetic stimulation devices. Brain Stimulation. 11(5), 1189-1191 (2018).

26. Lioumis, P., Zomorrodi, R., Hadas, I., Daskalakis, Z. J., Blumberger, D. M. Combined transcranial magnetic stimulation and electroencephalography of the dorsolateral prefrontal cortex. Journal of Visualized Experiments. (138), e57983 (2018)

27. Mishory, A. et al. The maximum-likelihood strategy for determining transcranial magnetic stimulation motor threshold, using parameter estimation by sequential testing is faster than conventional methods with similar precision. The Journal of ECT. 20(3), 160-165 (2004).

28. Thies, M., Zrenner, C., Ziemann, U., Bergmann, T. O. Sensorimotor mu-alpha power is positively related to corticospinal excitability. Brain Stimulation. 11(5), 1119-1122 (2018).

29. Schaworonkow, N. et al. $\mu$-Rhythm extracted with personalized EEG filters correlates with corticospinal excitability in real-time phasetriggered EEG-TMS. Frontiers in Neuroscience. 12, 954 (2018).

30. Hjorth, B. An on-line transformation of EEG scalp potentials into orthogonal source derivations. Electroencephalography and Clinical Neurophysiology. 39(5), 526-530 (1975).

31. Bergmann, T. O., Karabanov, A., Hartwigsen, G., Thielscher, A., Siebner, H. R. Combining non-invasive transcranial brain stimulation with neuroimaging and electrophysiology: Current approaches and future perspectives. Neurolmage. 140, 4-19 (2016). 